\title{
Anti-Nociceptive Synergism of Pregabalin and Xylopic acid Co-administration in Paclitaxel-induced Neuropathy: Isobolographic Analysis
}

\author{
Ameyaw Elvis Ofori ${ }^{1 *}$, Woode Eric ${ }^{3}$, Kyei Samuel ${ }^{2}$, Biny Peter Robert ${ }^{3}$ and Boampong Johnson Nyarko ${ }^{1}$ \\ 'Department of Biomedical and Forensic Sciences, School of Biological Sciences, College of Agriculture and Natural Sciences, University of Cape Coast, Cape Coast, \\ Ghana. \\ 2Department of Optometry, School of Physical Sciences, College of Agriculture and Natural Sciences, University of Cape Coast, Cape Coast, Ghana. \\ ${ }^{3}$ Department of Pharmacology, Faculty of Pharmacy and Pharmaceutical Sciences, College of Health Sciences, Kwame Nkrumah University of Science and Technology, \\ Kumasi, Ghana.
}

\begin{abstract}
Background: Paclitaxel-induced neuropathy still remains a clinical problem for patients undergoing chemotherapy. Objective: The objective of the study was to determine the interaction between xylopic acid and pregabalinco-administration as well as their toxicity in paclitaxel-induced neuropathy. Materials and Methods: Neuropathic pain was induced in rats with $2 \mathrm{mg} /$ $\mathrm{kg}$ of paclitaxel on four alternate days (days 0, 2, 4 and 6). Equi effective doses of xylopic acid and pregabalin that produced $50 \%$ anti-nociception $\left(E D_{50}\right)$ were determined from their log-dose response curves in the cold allodynia and thermal pain tests. Xylopic acid and pregabalin were again administered to rats in a fixed ratio combination $(1: 1)$ of their $E D_{50}$ 's in order to determine the experimental $\mathrm{ED}_{50}\left(\mathrm{Z}_{\text {exp }}\right)$ of the co-administered compounds. Isobolograms were constructed to compare the $Z_{\text {exp }}$ to a theoretical $E D_{50}$ $\left(Z_{\text {add }}\right)$. Blood samples from the various treatment groups of rats were collected for toxicological assessment of the co-administered compounds. Results: The $Z_{\text {exp }}$ lay below the $Z_{\text {add }}$ on the isobologram of the cold allodnia test. The co-administration exhibited additivity in the thermal pain test. The co-administration did not produce significant $(p>0.05)$ toxicity in rats. The co-administration may be beneficial in paclitaxel-induced neuropathy.
\end{abstract}

Key words: Isobolograms, Neuropathic pain, Paclitaxel, xylopic acid, Toxicity.

\section{SUMMARY}

- Paclitaxel-induced neuropathy is still a clinical problem.

- Pregabalin and Xylopic acid co-administration produced anti-nociceptive synergism against this pain type.

- The co-administration was fairly safe in animals.

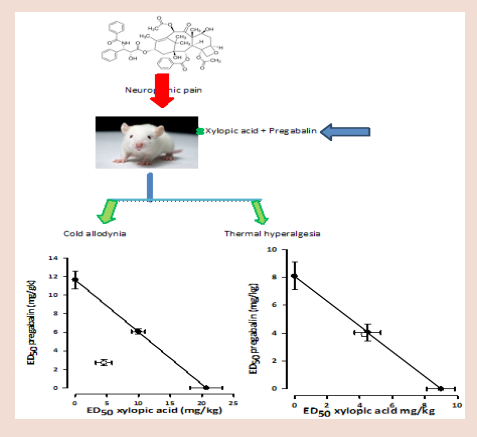

PICTORIAL ABSTRACT

Abbreviations used: XA: Xylopic acid, PG: Pregabalin, $Z_{\text {add }}$ : Theoritical $\mathrm{ED}_{50}, \mathrm{Z}_{\text {exp }}$ : Experimental $\mathrm{ED}_{50}$.

Correspondence:

Dr. Elvis Ofori Ameyaw, Department of Biomedical and Forensic Sciences, School of Biological Sciences, College of Agriculture and Natural Sciences, University of Cape Coast, Cape Coast, Ghana.

Phone no: +233279128631

Email: elvisameyaw@gmail.com

DOI : $10.5530 / p j .2015 .6 .8$

\section{INTRODUCTION}

Pain has been associated with a wide range of diseases such as cancer and arthritis. Pain can sometimes be the disease itself seen in conditions of neuropathy. ${ }^{1}$ Recently, it is reported that more than 1.5 billion people worldwide suffer from chronic pain. Again, approximately $3-4.5 \%$ of the global population suffers from neuropathic pain, with incidence rate increasing in complementary to age. ${ }^{2}$ Neuropathic pain arising from the cancer chemotherapeutic agent, paclitaxel still remains a clinical problem for patients undergoing chemotherapy. ${ }^{3}$ Paclitaxel is one of the most effective and commonly used anticancer drugs for the treatment of solid tumours. Paclitaxel produces neuropathy by destroying the nerves via its binding to $\beta$-tubulin of microtubules. This impairs axoplasmic transport leading to a progressive dying-back axonopathy, hence the neuropathy. Evidence of swollen and vacuolated mitochondria in peripheral sensory axons, both C-fibers and myelinated axons has been reported. ${ }^{4}$ Its use is limited by Myelossupression and peripheral neurotoxicity. ${ }^{3}$ Clinically, the sensory neurotoxicity produced by paclitaxel include allodynia, numbness, tingling, and burning pain which often begin symmetrically in the feet and sometimes appear simultaneously in both hands and feet. The neuropathic pain sometimes becomes a chronic problem even after cessation of paclitaxel administration. ${ }^{5}$ Despite this problem confronted by patients, there is currently no valid drug to prevent or treat the paclitaxel-induced pain. ${ }^{6}$ Pregabalin is the only drug currently approved in the USA for the treatment of spinal neuropathic pain, a type of pain condition affecting $40 \%$ of spinal cord injury patients. ${ }^{7}$ There is the likelihood for food-drug interaction occurring between xylopic acid and pregabalin especially in Africa. Patients prescribed with pregabalin for their disease conditions may ingest xylopic acid which is present in the fruits of Xylopia aethiopica. Xylopia aethiopica is consumed as a delicacy in most parts of the world. ${ }^{8}$ This presents important drug-food interaction because xylopic acid is the highest occurring compound in the fruits of $X$. aethiopica. ${ }^{9}$ Xylopic acid has demonstrated antinociceptive property in several models of pain. The relief of pain by xylopic acid has been attributed to its action on the opioidergic, adrenergic, bradykinin and prostaglandin nociceptive pathways. ${ }^{10-12}$ Pregabalin, an antiepileptic drug has demonstrated analgesic property in paclitaxel-induced neuropathic pain in pre-clinical, clinical research and therapy. ${ }^{13}$ 
To improve the analgesic potency and efficacy of xylopic acid and pregabalin against paclitaxel-induced neuropathy in rats, isobolographic study was performed on these two agents. Because the effects of co-administered drugs are often highly variable, such variability necessitates the use of statistical robust methodology. Isobolographic study is a tool that provides unambiguous terminologies of drug interactions and a statistical framework for the analysis of drug combinations or co-administration. Drug combination regimens have been utilised in areas of HIVAIDS, cancer and tuberculosis with the aim of achieving a therapeutic efficacy greater than that achieved with monotherapy with some success. Other benefits include decreased toxicity-antagonistic interaction, the delay or prevention of drug resistance development, and the favourable effects of synergistic drug interactions. Synergism is especially important in clinical situations with drugs because it allows the use of smaller amounts of the constituent drugs. An adverse effect may also synergise, presenting a phenomenon of special importance in clinical situations which often leads to reduction of doses of the individual drugs for therapy. The detection of synergism may also be useful in illuminating mechanism of drug action and in the development of new theories. ${ }^{14,15}$ It is therefore possible to upsurge the analgesic efficacy and potency of xylopic acid and pregabalin and correspondingly decrease their side effects via combination therapy.

\section{METHODS AND MATERIALS}

\section{Extraction of xylopic acid (15 $\beta$-Acetoxy(-)-kaur-16-en-19-oic} acid)

Xylopic acid was extracted according to the process described elsewhere. ${ }^{9,10}$ Briefly, $360 \mathrm{~g}$ of dried fruit of Xylopia aethiopica was macerated with $5 \mathrm{~L}$ of petroleum ether $\left(40-60^{\circ} \mathrm{C}\right)$. This was and allowed to stand for three days. The petroleum ether was drained and concentrated with rotary evaporator at a temperature of $50^{\circ} \mathrm{C}$. Crude xylopic acid formed after three days was washed with petroleum ether at $40-60^{\circ} \mathrm{C}$ and purified with $96 \%$ ethanol. The yield of the xylopic acid was $1.41 \%$, melting point of $261-262^{\circ} \mathrm{C}$. The purity of the extracted xylopic acid as determined with High Performance Liquid Chromatography was 95\% as described previously. ${ }^{9}$

\section{Animals and husbandry}

Sprague-Dawley rats (150-200 g) of both sexes were housed in the animal facility of the Department of Biomedical and Forensic Sciences. The animals were housed in groups of six in stainless steel cages $(34 \times 47 \times 18$ $\mathrm{cm}$ ) with soft wood shavings as bedding. They were fed with normal commercial pellet diet (AGRICARE, Kumasi, Ghana), given water ad libitum and maintained under standard laboratory conditions. All procedures and techniques used in these studies were in accordance with the National Institute of Health Guidelines for the Care and Use of Laboratory Animals. All protocols used were approved by the Departmental Ethics Committee.

\section{Drugs and reagents}

Pregabalin $\left(\right.$ Lyrica $\left.^{\oplus}\right)$ was purchased from Pfizer Pharmaceuticals, Arzneimittelwerk Godecke, Freiburg, Germany, cremophor from SigmaAldrich Inc., St. Louis, MO, USA and paclitaxel (Intaxel ${ }^{\circledR}$ ) from Fresinius Kabi Oncology, Badi, India.

\section{Paclitaxel administration}

Baseline measurements of thermal and cold stimuli were performed after allowing the rats to acclimatise to the behavioural testing environment. Neuropathic pain was induced in the rats by intraperitoneal (i.p.) injection of paclitaxel $(2 \mathrm{mg} / \mathrm{kg})$ dissolved in saline on four alternate days (days $0,2,4$ and 6) as described. ${ }^{16}$ On day 16 post- paclitaxel administra- tion, the various groups of animals were treated with vehicle (cremophore solution) xylopic acid (10-100 $\mathrm{mg} / \mathrm{kg}$ in cremophore) and pregabalin $(10-100 \mathrm{mg} / \mathrm{kg})$ for five days.

\section{Cold allodynia}

The analgesic effect of orally co-administered xylopic acid and pregabalin on cold allodynia was assessed by immersing the rat's hind paw into cold water $\left(4.5^{\circ} \mathrm{C}\right)$. The latency for a rat to withdrawal its paw was measured with a digital timer. ${ }^{17}$ Just one hind paw was assessed during each immersion at a time with a cut-off time of $20 \mathrm{~s}$. For each animal, two recordings were made for each hind paw, and the withdrawal responses were reported as the mean of both hind paw values. The treatment was continued with vehicle, xylopic acid and pregabalin for 5 days.

\section{Thermal Hyperalgesia}

The tail immersion test was done by immersing the distal portion of the tail $(3-4 \mathrm{~cm})$ of the various treated groups of rats in hot water maintained at $52^{\circ} \mathrm{C}$ until the tail was withdrawn. The duration of immersion was recorded and a cut-off time of $10 \mathrm{~s}$ was used. ${ }^{18}$ The treatment was continued with vehicle, xylopic acid and pregabalin for 5 days.

Isobolographic analysis of analgesic property and toxicological assessment of xylopic acid and pregabalin co-administration

The potencies of xylopic acid and pregabalin estimated previously in the cold allodynia and thermal hyperalgesia tests were used for the isobolographic studies. The estimated potencies $\left(\mathrm{ED}_{50}\right.$ 's) of xylopic acid and pregabalin in both tests were also used to compute the theoretical potency (Zadd) as follows;

$$
\text { Zadd }=f\left(E D_{50}\right) \text { of pregabalin }+(1-f) E D_{50} \text { of xylopic acid }
$$

Where $f$ is the fraction of the each component in the mixture. ${ }^{15}$

To obtain the combination potency of co-administered xylopic acid (XA) and pregabalin (PG), the two agents were orally administered to paclitaxel-induced neuropathic pain suffering rats daily at doses of their respective $\mathrm{ED}_{50}$ 's and in fixed ratio combinations of fractions of their respective $\mathrm{ED}_{50}$ values (equieffctive doses) of $1 /{ }_{2}, 1 / /_{4}, 1 /{ }_{8}, 1 /{ }_{16}$. The treatment regime continued for 5 days in the cold allodynia and thermal hyperalgesia tests. The experimental potency $\left(\mathrm{Z}_{\mathrm{exp}}\right)$ of the co-administered test agents $(\mathrm{XA}+\mathrm{PG})$ was determined by least square method of regression.

To determine the drug-drug interaction in the living system, isobologram (a cartesian plot of pairs of doses that, in combination, yield a specified level of effect) was constructed by connecting the theoretical $\mathrm{ED}_{50}$ of xylopic acid plotted on the abscissa and pregabalin plotted on the ordinate to obtain the line of additivity. The $\mathrm{ED}_{50}$ (experimental) was compared to the theoretical additive $\mathrm{ED}_{50}$ using a ' $t$ '-test. Synergistic analgesic effect is obtained when the effect of a drug combination is higher and statistically different $\left(\mathrm{ED}_{50}\right.$ significantly lower) than the theoretically calculated equieffective dose of a drug combination with the same proportions. If the $\mathrm{ED}_{50}$ 's are not statistically different, the effect of the combination is additive which implies each constituent contributes with its own potency to the total effect. The interaction index was also computed as $\mathrm{Z}_{\exp } / \mathrm{Z}_{\text {add }}$. If the value is close to one, the interaction is additive. Values lower than one indicates the magnitude of synergistic interactions and values higher than 1 represent antagonistic interactions. Blood samples were also collected for haematology and biochemical assay for signs of toxicity on the fifth day post-treatment. The haematological parameters were determined with an automatic analyzer (Sysmex XT-2000lCELLDYN 1700, Abbot Diagnostics Division, Abbot Laboratories, Abbot Park, Illinois, USA). Haematological parameters estimated included haemoglobin (Hb), erythrocyte count (RBC), haematocrit (HCT), mean corpuscular volume (MCV), mean corpuscular haemoglobin $(\mathrm{MCH})$, mean corpuscular haemoglobin concentration $(\mathrm{MCHC})$, platelet count 


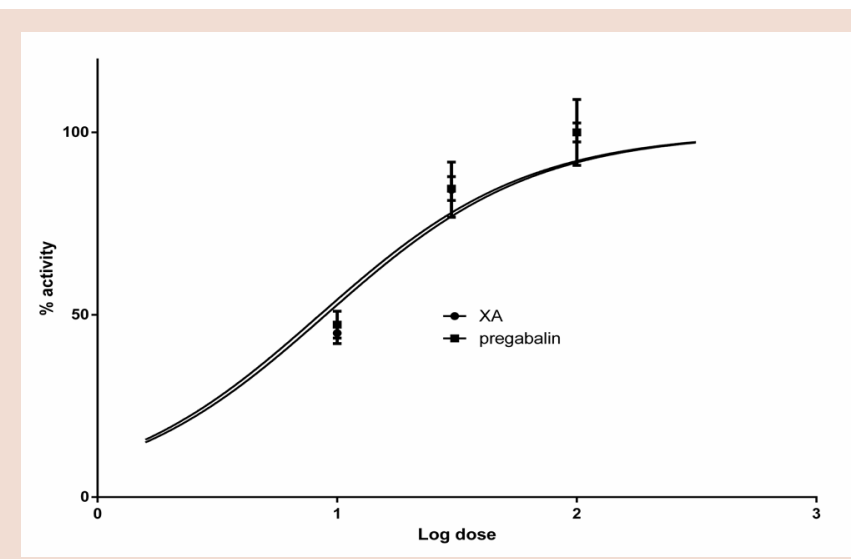

Figure 1: Dose-response curves of (a) xylopic acid and (b) pregabalin in the tail immersion test

Data is presented as mean \pm S.E.M

(PLT), and total white blood cells (WBCs). The serum biochemistry parameters studied were alanine aminotransferase (ALT), aspartate aminotransferase (AST), alkaline phosphatase (ALP), gamma glutamyl transferase (GGT), bilirubin, blood urea nitrogen (BUN), creatinine, total protein (protein) and albumin.

\section{Statistical analysis}

The data were analysed with GraphPad Prism Version 5 (GraphPad Software, San Diego, CA, USA). The results are presented as mean \pm S.E.M. The difference between the means of co-treated groups and negative control group was analysed with One-way analysis of variance followed by Tukey's post hoc test. Doses for $50 \%$ of the maximal effect $\left(\mathrm{ED}_{50}\right)$ for each drug were determined using an iterative computer least squares method, with the following nonlinear regression (three-parameter logistic) equation:

$$
Y=\frac{a+(b-a)}{\left(1+10^{\left({\left.\log E D_{50}-X\right)}\right)}\right.}
$$

Where, $\mathrm{X}$ is the logarithm of dose and $\mathrm{Y}$ is the response. $\mathrm{Y}$ starts at a (the bottom) and goes to $\mathrm{b}$ (the top) with a sigmoid shape. $\mathrm{P}<0.05$ was considered statistically significant. The fitted midpoints $\left(E_{50} s\right)$ of the curves, i.e., $\mathrm{Z}_{\exp }$ and $\mathrm{Z}_{\text {add }}$ were compared statistically using t-test. ${ }^{19}$ Isobolographic calculations were performed with the program Pharm Tools Pro (version 1.27, the Mc Cary Group Inc.). Results are presented as mean \pm S.E.M.

\section{RESULTS}

Estimation of the potencies of xylopic acid and pregabalin in the cold allodynia and thermal hyperalgesia tests

The potencies of xylopic acid and pregabalin were $8.97 \pm 0.96 \mathrm{mg} / \mathrm{kg}$ and $8.1 \pm 0.85 \mathrm{mg} / \mathrm{kg}$ in the thermal pain tests respectively (Figure 1). In the cold allodynia test, the $\mathrm{ED}_{50}$ of xylopic acid and pregabalin were $20.64 \pm$ $0.99 \mathrm{mg} / \mathrm{kg}$ and $11.65 \pm 095 \mathrm{mg} / \mathrm{kg}$ respectively (Figure 2). The fractions of the $\mathrm{ED}_{50}$ of xylopic acid and pregabalin used for the isobolographic studies are shown on Table 1.

Isobolographic assessment of the analgesic effect of xylopic acid and pregabalin co-administration in the cold allodynia and thermal hyperalgesia tests

The co-administration of XA and PG resulted in significant analgesic effect compared to the vehicle treated animals. The theoretical $\mathrm{ED}_{50}$ 's in the

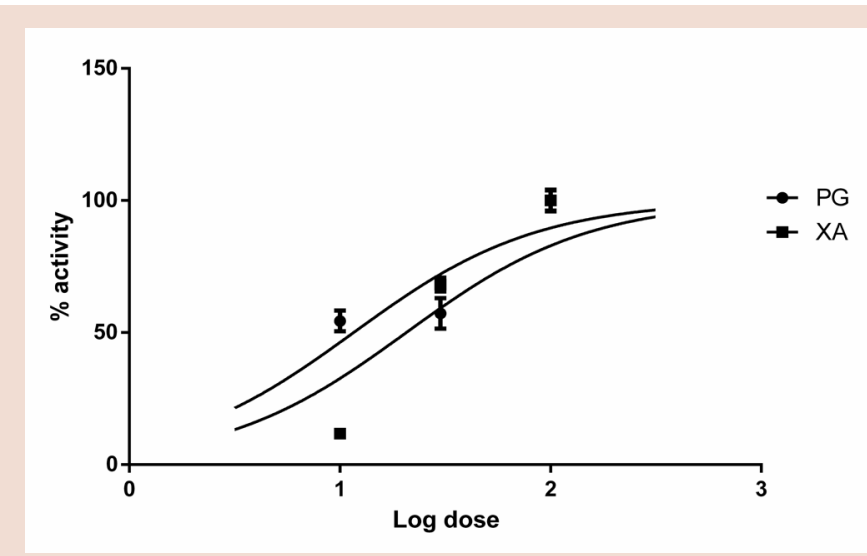

Figure 2: Dose-response curves of xylopic acid and pregabalin in the cold allodynia test

Data is presented as mean \pm S.E.M

cold allodynia and thermal hyperalgesia tests were $16.15 \pm 1.2$ and $8.54 \pm$ 1.4 respectively. The experimental $\mathrm{ED}_{50}(\mathrm{Zexp})$ for the mixture $(\mathrm{XA}+\mathrm{PG})$ in the cold allodynia and thermal pain tests were $7.25 \pm 0.23$ and $8.16 \pm$ 1.6 respectively. The Zexp (open circle) lay significantly below the line of additivity and the Zadd (closed circles) on the isobologram of the cold allodynia but not the thermal hyperalgesia test (Figure $3 \mathrm{a}$ and $3 \mathrm{~b}$ ) indicating synergism of analgesic effect $(\mathrm{P}<0.001)$ in the cold allodynia test but not the thermal pain test. The degree of interaction calculated as the interaction indices were 0.45 and 0.96 for the cold and thermal tests respectively (Table 2).

Effect of xylopic acid and pregabalin co-administration on haematological parameters and heart, liver and kidney enzymes

The vehicle treated group and XA+PG co-treated groups had reduced but not significant white blood cells concentration compared to sham control groups (Table 3). The liver and bile enzymes, AST, ALT, GGT and ALP from the animals co-administered with various concentrations of XA and PA combination were not statistically different from the negative control groups. Albumin and total protein concentrations were also not different from the negative control group. The ALT, ALP and AST concentrations of the vehicle treated group were slightly elevated but not statistically significant from that of the sham group (Table 3). The kidney

Table 1: The $\mathrm{ED}_{50}$ 'S \pm S.E.M. and fractions of Xylopic acid and Pregabalin used for determining the $Z_{\text {exp }}$ in the cold allodynia and thermal hyperalgesia tests

\begin{tabular}{ccc}
\hline & \multicolumn{2}{c}{ Doses used $(\mathrm{mg} / \mathrm{kg})$} \\
\hline Tests & Xylopic acid & Pregabalin \\
& $8.97 \pm 0.96$ & $8.1 \pm 0.85$ \\
Thermalpain & 4.49 & 4.05 \\
(Tail immersion test) & 2.24 & 2.025 \\
& 1.12 & 1.013 \\
& 0.56 & 0.506 \\
Cold allodynia & $20.64 \pm 0.99$ & $11.65 \pm 0.95$ \\
& 10.32 & 5.825 \\
& 5.16 & 2.913 \\
& 2.85 & 1.456 \\
& 1.29 & 0.728 \\
\hline
\end{tabular}




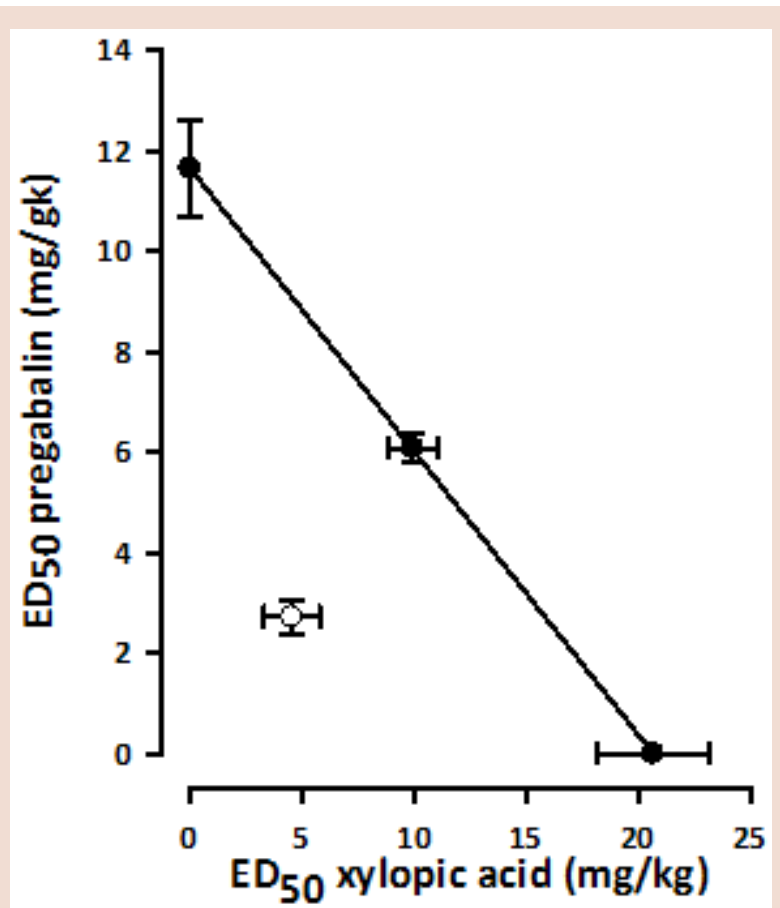

Figure 3a: Isobologram for the combination of xylopic acid and pregabalin in (a) cold allodynia test in rats. Filled circles $(\bullet)$ are the theoretical ED50's \pm S.E.M. and open circles (o), the experimental ED50's \pm S.E.M

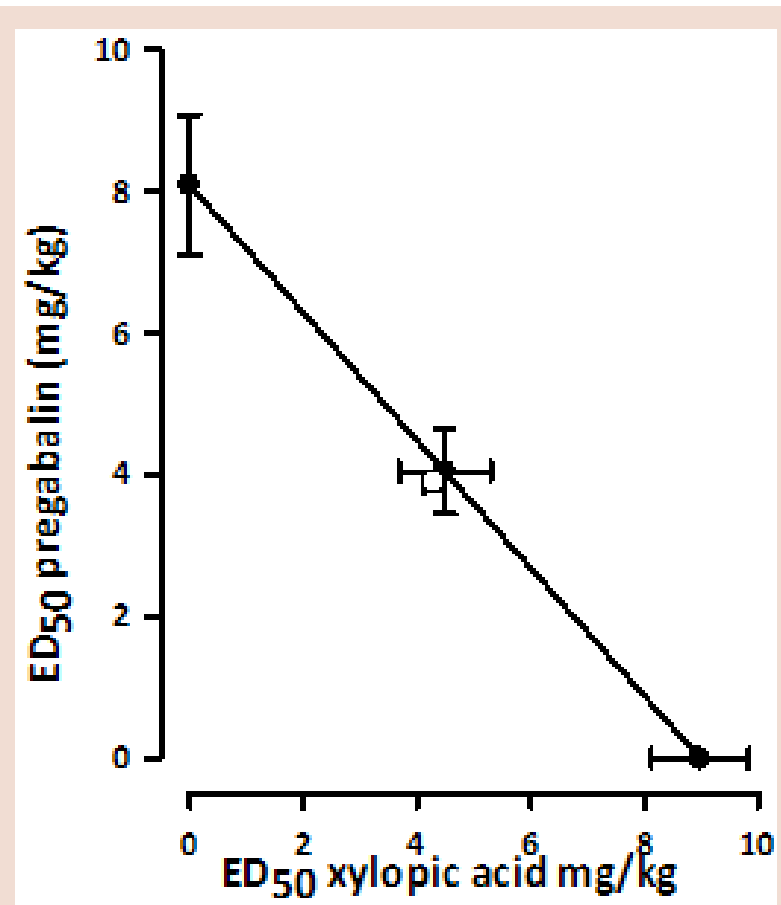

Figure 3b: Isobologram for the combination of xylopic acid and pregabalin in (b) thermal hyperalgesia tests in rats. Filled circles $(\bullet)$ are the theoretical ED50's \pm S.E.M. and open circles (o), the experimental ED50's \pm S.E.M

\begin{tabular}{|c|c|c|}
\hline \multicolumn{3}{|c|}{ Tests } \\
\hline$E D_{50}{ }^{\prime} s(X A+P G: 1: 1)$ & $\begin{array}{c}\text { Thermal pain (Tail immersion } \\
\text { test) }\end{array}$ & Cold allodynia \\
\hline Zadd (mg/kg) & $8.54 \pm 1.4$ & $16.15 \pm 1.2$ \\
\hline$Z \exp (\mathrm{mg} / \mathrm{kg})$ & $8.16 \pm 1.6$ & $7.25 \pm 0.23^{.+* x}$ \\
\hline Interaction index & 0.96 & 0.45 \\
\hline
\end{tabular}

Values are presented as Mean \pm S.E.M. ${ }^{* * x} \mathrm{P}<0.001$ compared to their respective $\mathrm{Z}_{\text {adds }}$

enzymes and function marker, creatinine and BUN were insignificantly altered in the $\mathrm{XA}+\mathrm{PG}$ co-treated groups compared to the vehicle treated group (Table 3 ). The various co-treatments did not produce toxicity on the erythrocytes and their contents (Table 3).

\section{DISCUSSION}

Oral co-administration of pregabalin and xylopic acid produced synergistic analgesic properties and no significant toxic effects in rat model of paclitaxel-induced neuropathic pain. The potency of co-administered agents, xylopic acid and pregabalin was significantly higher, and lay below the line of additivity and the theoretical potency of the two agents in the cold allodynia test. The additivity line represents the level of effect for the individual agents and the closeness of the experimental potency of the mixture to this line in the thermal pain test demonstrates additivity. ${ }^{20}$ Both compounds were more potent in the thermal pain test compared to the cold allodynia test. On the contrary, the magnitude of potentiation, with reference to the interaction indices was greater in the cold allodynia test than the thermal pain test. The difference in efficacy of the co-administered agents in the two tests may be due to the different pathophysiology associated with the two pain types. It is obvious that the efficacy of the two agents as well as the co-administration vary with respect to type of noxious stimulus.
Paclitaxel, after its administration accumulates in the dorsal root ganglia and the brain at very low concentrations. Accumulation has also been reported in the sciatic nerve and spinal cord at intermediate concentrations. ${ }^{21}$ Morphological changes such as swollen and vacuolated mitochondria as well as reduced respiration and energy production in axonal mitochondria that results from paclitaxel administration initiates apoptosis pathways by altering intracellular calcium levels. ${ }^{4,22}$ Therefore, the neuropathy in this study after low dose paclitaxel administration was due to atypical (swollen and vacuolated) mitochondria in peripheral sensory axons, both C-fibers and myelinated axons as well as the loss of intraepidermal nerve fibres. Both agents may have acted centrally to produce analgesic synergism. This is supported by the tail immersion test. The tail immersion thermal pain test investigates pain primarily of spinal origin. ${ }^{23}$ Pregabalin is a well-established anticonvulsant and analgesic agent that has shown efficacy and dose dependent effects either as monotherapy or in combination with analgesics in relieving pain and related symptoms. It is noteworthy that pregabalin has received Food and Drug Association (FDA) approval for the treatment of diabetic neuropathy and post-herpetic neuralgia. Pregabalin produces its analgesic effect through high-affinity alpha2-delta-1 subunit of voltage-gated calcium channels. Both xylopic acid and pregabalin are calcium channel blockers 
Table 3: Effect of Xylopic acid and Pregablin co-administration on blood biochemistry and haematological parameters

\begin{tabular}{|c|c|c|c|c|c|c|c|}
\hline \multirow{2}{*}{ Parameter } & \multirow{2}{*}{ Sham } & \multirow{2}{*}{ NC } & \multicolumn{5}{|c|}{ Doses of XA and PG $(\mathrm{mg} / \mathrm{kg})$} \\
\hline & & & $X A+P G$ & $X A+P G / 2$ & $X A+P G / 4$ & $X A+P G / 8$ & $X A+P / 16$ \\
\hline BUN (mg \%) & $38.4 \pm 0.5$ & $39.1 \pm 0.2$ & $39.3 \pm 0.6$ & $38.9 \pm 0.2$ & $39.2 \pm 0.5$ & $38.8 \pm 0.4$ & $38.5 \pm 0.5$ \\
\hline $\begin{array}{c}\text { Creatinine } \\
(\mathrm{mg} / \mathrm{dl})\end{array}$ & $29.4 \pm 1.1$ & $30.5 \pm 2.5$ & $31.4 \pm 0.7$ & $30.7 \pm 1.5$ & $29.5 \pm 1.8$ & $30.5 \pm 1.1$ & $30.2 \pm 1.8$ \\
\hline $\begin{array}{l}\text { Total bilirubin } \\
\text { (mg/dl) }\end{array}$ & $0.96 \pm 0.1$ & $1.1 \pm 0.2$ & $1.1 \pm 0.9$ & $1.1 \pm 0.1$ & $1.1 \pm 0.2$ & $1.0 \pm 0.2$ & $1.0 \pm 0.5$ \\
\hline $\operatorname{ALP}(\mathrm{IU} / \mathrm{l})$ & $0.7 \pm 0.8$ & $0.8 \pm 0.1$ & $0.9 \pm 0.4$ & $0.9 \pm 0.2$ & $0.8 \pm 0.1$ & $0.9 \pm 0.3$ & $0.8 \pm 0.1$ \\
\hline ALT(IU/l) & $195 \pm 3.4$ & $193 \pm 1.8$ & $200 \pm 2.5$ & $163 \pm 9.7$ & $187 \pm 4.3$ & $189 \pm 1.2$ & $191 \pm 2.4$ \\
\hline AST/(IU/l) & $59.5 \pm 1.2$ & $60.5 \pm 0.5$ & $68.2 \pm 0.2$ & $66.5 \pm 1.4$ & $59.8 \pm 0.1$ & $59.9 \pm 1.5$ & $59.7 \pm 1.2$ \\
\hline GGT(IU/l) & $150 \pm 1.3$ & $153 \pm 1.4$ & $152 \pm 1.2$ & $150 \pm 1.8$ & $145 \pm 0.5$ & $144 \pm 0.5$ & $150 \pm 1.3$ \\
\hline Protein $(\mathrm{g} / \mathrm{dl})$ & $11.4 \pm 1.4$ & $12.4 \pm 0.3$ & $12.1 \pm 1.5$ & $10.1 \pm 1.3$ & $7.2 \pm 0.5$ & $9.4 \pm 1.5$ & $10.5 \pm 0.2$ \\
\hline Albumin $(g / l)$ & $75.4 \pm 0.5$ & $77.5 \pm 0.8$ & $74.2 \pm 0.4$ & $75.3 \pm 1.5$ & $71.2 \pm 1.6$ & $77.4 \pm 0.9$ & $74.3 \pm 0.8$ \\
\hline $\mathrm{WBC}\left(\times 10^{3} / \mu \mathrm{L}\right)$ & $40.2 \pm 0.8$ & $42.3 \pm 0.4$ & $39.5 \pm 08$ & $42.1 \pm 0.4$ & $43.2 \pm 1.2$ & $42.1 \pm 0.9$ & $40.3 \pm 0.5$ \\
\hline $\begin{array}{l}\text { Platelets } \\
\left(\times 10^{3} / \mu \mathrm{l}\right)\end{array}$ & $13.2 \pm 0.9$ & $11.9 \pm 0.9$ & $11.1 \pm 0.4$ & $12.2 \pm 0.1$ & $13.5 \pm 1.1$ & $14.95 \pm 0$ & $14.95 \pm 0$ \\
\hline $\mathrm{RBC}\left(\times 10^{6} / \mu \mathrm{L}\right)$ & $301 \pm 1.3$ & $298 \pm 1.1$ & $305 \pm 0.9$ & $308 \pm 1.5$ & $298 \pm 1.1$ & $304 \pm 0.8$ & $309 \pm 01.3$ \\
\hline HCT (\%) & $8.5 \pm 2.5$ & $8.9 \pm 1.3$ & $8.7 \pm 2.3$ & $9.2 \pm 0.7$ & $8.1 \pm 1.2$ & $8.5 \pm 0.65$ & $8.8 \pm 1.3$ \\
\hline $\mathrm{Hb}(\%)$ & $34.1 \pm 1.2$ & $32.2 \pm 1.5$ & $30.1 \pm 1.5$ & $32.8 \pm 0.8$ & $35.5 \pm 0.9$ & $30.9 \pm 1.3$ & $33.8 \pm 1.2$ \\
\hline MCH (pg) & $12.2 \pm 0.4$ & $12.7 \pm 0.5$ & $13.7 \pm 1.1$ & $10.3 \pm 0.9$ & $12.5 \pm 0.2$ & $12.4 \pm 0.9$ & $10.9 \pm 1.2$ \\
\hline $\operatorname{MCV}\left(\mu \mathrm{m}^{3}\right)$ & $30.4 \pm 0.5$ & $28.4 \pm 1.2$ & $26.4 \pm 0.8$ & $28.4 \pm 1.2$ & $25.6 \pm 0.5$ & $29.4 \pm 0.9$ & $29.1 \pm 1.4$ \\
\hline MCHC (\%) & $54.8 \pm 1.2$ & $53.4 \pm 0.9$ & $50.2 \pm 0.8$ & $54.3 \pm 0.2$ & $51.8 \pm 1.2$ & $56.1 \pm 0.9$ & $56.1 \pm 1.4$ \\
\hline
\end{tabular}

$\mathrm{NC}=$ Negative Control.

with the ability to stabilise injured nerve membrane. ${ }^{24,25}$ This mechanism may underline the analgesic effect of the co-administered agents in the thermal pain test because additivity is at best observed when two agents activate the same pathway. ${ }^{15}$

Xylopic acid produces analgesic effect in neuropathic pain by acting on NMDA and adrenergic nociceptive pathways. ${ }^{12}$ Any of these nociceptive pathways might have been modulated by xylopic acid in addition to the inhibition of $\mathrm{Ca}^{2+}$ channel-mediated neurotransmitter release, activation of excitatory amino acid transporters, modulation of potassium channels and inhibition of pathways involving inflammatory mediators by pregabalin to produce the observed synergism in the cold allodynia test. ${ }^{24}$ Although xylopic acid and pregabalin co-administration produced analgesic synergism and additivity, the combination may not be without toxic effects, especially on prolong usage. Paclitaxel slightly reduced the red blood cells and platelets count and these effects were reversed by the co-administered agents. Paclitaxel as well as the coadministered agents may have the potential to induce hepato-biliary diseases on prolong usage. Generally, a slight elevation of ALP and total bilirubin was observed for paclitaxel and the relative high dose combination treatments. The liver enzymes were not significantly affected by the co-administration.

It is also likely that the long term use of the co-administered agents will have deleterious effect on the kidneys. Blood urea nitrogen and serum creatinine were slightly but not significantly elevated in the paclitaxel and co-administered agents treated groups. This may indicate the diminished excretory efficiency of the kidneys of these animals. ${ }^{26}$ This implies the co-administration of xylopic acid and pregabalin in neuropathic pain states associated with paclitaxel cancer chemotherapy is fairly safe. However, further studies to determine the long term safety of the co-administration is needed to exclude toxicity from cumulative doses.

\section{CONCLUSION}

Co-administration of xylopic acid and pregabalin may be beneficial in some types of painful neuropathy associated with paclitaxel administration. The co-administration is also fairly safe.

\section{ACKNOWLEDGEMENTS}

The authors express their sincere gratitude to Mr. Kwame Kumi Asare, Daniels Konja and Rex Annane Frimpong. Department of Biomedical and Forensic Sciences, University of Cape Coast.

\section{CONFLICT OF INTEREST}

The authors declare no conflict of interest.

\section{REFERENCES}

1. Hansson P. Neuropathic pain: clinical characteristics and diagnostic workup European Journal of Pain 2002; 6(SA): 47-50.

2. Global Industry Analysts, Inc. Report, January 10, 2011. http://www.prweb.com/ pdfdownload/8052240.pdf.

3. Rowinsky EK, Eisenhauer EA, Chaudhry V, Arbuck SG, Donehower RC. Clinical toxicities encountered with paclitaxel (Taxol). Semin Oncol. 1993; 20(s3): 1-15.

4. Xiao WH, Zheng H, Zheng FY, Nuydens R, Meert TF, Bennett GJ. Mitochondrial abnormality in sensory, but not motor, axons in paclitaxel-evoked painful peripheral neuropathy in the rat. Neuroscience 2011; 199(2011): 461-9.

5. Polomano RC, Mannes AJ, Clark US, Bennett GJ. A painful peripheral neuropathy in the rat produced by the chemotherapeutic drug, paclitaxel. Pain 2001; 94(3): 293-304.

6. Liu CC, Lu N, Cu Yi, Yang T, Zhao ZQ, Xin WJ, et al. Prevention of Paclitaxelinduced allodynia by Minocycline: Effect on loss of peripheral nerve fibers and infiltration of macrophages in rats. Molecular Pain 2010; 6(1): 76.

7. Pfizer Inc. (NYSE: PFE). FDA Approves Lyrica For The Management Of Neuropathic Pain Associated With Spinal Cord Injury Based On Priority Review. New york-business wire 2012. http://press.pfizer.com/press-release/fda-approveslyrica-management-neuropathic-pain-associated-spinal-cord-injury-based-pr. 
8. Woode E, Alhassan A, Abaidoo CS. Effect of ethanolic fruit extract of Xylopia aethiopica on reproductive function of male rats. Int J Pharm Biomed Res. 2011; 2(3): 161-5.

9. Adosraku RK, Oppong- Kyekyeku J. Characterization and HPLC quantification of xylopic acid in the dried fruits of Xylopia aethiopica. Int. J. Pure Applied Chem. $2011 ; 6(2): 209-13$

10. Ameyaw EO, Boampong JN, Kukuia KE, Amoateng P, Obese E, Osei-Sarpong $\mathrm{C}$, et al. Effect of xylopic acid on paclitaxel-induced neuropathic pain in rats. Journal of Medical and Biomedical Sciences 2013; 2(4): 6-12.

11. Woode E, Ameyaw EO, Boakye-Gyasi E, Abotsi WKM. Analgesic Effects of an Ethanol Extract of the Fruits of Xylopia aethiopica (Dunal) A. Rich. (Annonaceae) and the Major Constituent, Xylopic Acid in Murine Models. Journal of Pharmacy and Bioallied Sciences 2012; 4(4): 301-429.

12. Woode E, Ameyaw EO, Ainooson GK, Abotsi WKM, Boakye-Gyasi E, OppongKyekyeku J. Analgesic Effects of an Ethanol Extract of the Fruits of Xylopia aethiopica and xylopic acid in Murine Models: Possible Mechanism(s). Pharmacologia 2013; 4(4): 285-300

13. Chogtu B, Bairy KL, Smitha D, Supurna D, Himabindu P. Comparison of the efficacy of carbamazepine, gabapentin and lamotrigine for neuropathic pain in rats. Indian J Pharmacol. 2011; 43(5): 596-8.

14. Fivelman LQ, Adagu IS, Warhurst DC. Modified fixed-ratio isobologram method for studying in vitro interactions between atovaquone and proguanil or dihydroartemisinin against drug-resistant strains of Plasmodium falciparum. Antimicrob Agents Chemother. 2004; 48(11): 4097-102.

15. Tallarida RJ. An overview of drug combination analysis with isobolograms. J Pharmacol Exp Ther. 2006; 319(1): 1-7

16. Flatters SJ, Bennett GJ. Studies of peripheral sensory nerves in paclitaxel induced painful peripheral neuropathy: evidence for mitochondrial dysfunction. Pain 2006: 122(3): 245-57.
17. Kim SK, Park JH, Bae SJ, Bae SJ, Kim JH, Hwang BG, et al. Effects of electro acupuncture on cold allodynia in a rat model of neuropathic pain: mediation by spinal adrenergic and serotonergic receptors. Exp Neurol. 2005; 195(2): 430-6.

18. Thirumal M, Srimanthula S, Kishore G, Vadivelan R, Anand Kumar AVS. Analgesic and antipyretic effects of aqueous extract from Clerodendrum inerme (L.) Gaertn. leaves in animal models. Der Pharmacia Lettre 2011; 5(2): 315-23.

19. Motulsky HJ, Christopoulos A. Fitting model to biological data using linear and nonlinear regression. A practical guide to curve fitting. San Diego, CA, Graph Pad Software Inc; 2003

20. Ugalde M, Reza V, Gonza' lez-Trujano ME, Avula B, Khan IA, Navarrete A. Isobolographic analysis of the sedative interaction between six central nervous system depressant drugs and Valeriana edulis hydroalcoholic extract in mice. Journal of Pharmacy and pharmacology 2005; 57(5): 631-9.

21. Cavaletti G, Cavalletti E, Oggioni N, Sottani C, Minoia C, D'Incalci M, et al Distribution of paclitaxel within the nervous system of the rat after repeated intravenous administration. Neurotoxicology 2000; 21(3): 389-93.

22. Fidanboylu M, Griffiths LA, Flatters SJL. Global Inhibition of Reactive Oxygen Species (ROS) Inhibits Paclitaxel-Induced Painful Peripheral Neuropathy. plos one. 2011; 6(9): e25212

23. Adzu B, Amos S, Kapu SD, Gamaniel KS. Anti-inflammatory and antinociceptive effects of Sphaeranthus senegalensis. J. Ethnopharmacol. 2003; 84(2): 169-73.

24. Verma V, Singh N, Singh Jaggi A. Pregabalin in Neuropathic Pain: Evidences and Possible Mechanisms. Current Neuropharmacology. 2004; 12(1): 44-56.

25. Somova LI, Shode FO, Moodley K, Govender YJ. Cardiovascular and diuretic activity of kaurene derivatives of Xylopia aethiopica and Alepidea amatymbica. Ethnopharmacol. 2001; 77(2): 165-74.

26. Whelton A, Watson AY, Rock RC. In: Burtis, CA, Ashwood ER. Tietz Text book of Clinical Chemistry. WB. Saunders Company, London; 2004. 1528-31.

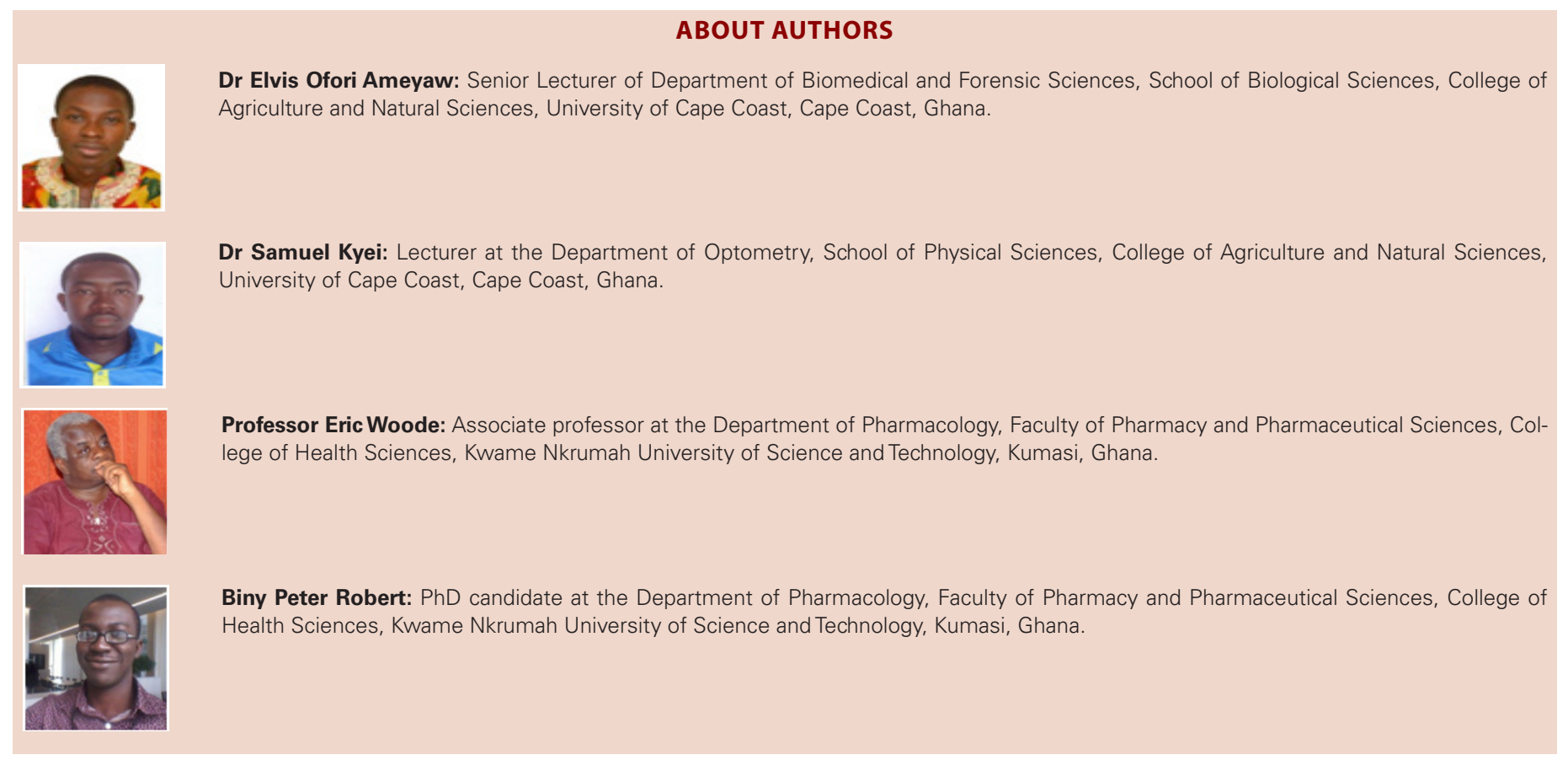

